\title{
Dynamo - software for vibration based Structural Health Monitoring
}

\author{
F. Magalhães, S. Amador, Á. Cunha \& E. Caetano \\ University of Porto, Faculty of Engineering (FEUP), Porto, Portugal
}

\begin{abstract}
This paper presents an innovative software for continuous dynamic monitoring of civil infrastructures. The followed approach is based in the continuous on-line automatic identification of the structure modal parameters, using its response under operation and adopting state-of-the-art identification algorithms. Therefore, the monitoring software, called DynaMo, includes routines for data and results management, algorithms for operational modal analysis, statistical tools for elimination of environmental and operational factors on the identified modal parameters and also statistical tools for automatic identification of abnormal frequency values that might be associated with the occurrence of damages. The utility and efficiency of DynaMo is illustrated with an application on a large span concrete arch bridge that is being monitored since 2007.
\end{abstract}

\section{INTRODUCTION}

A Structural Health Monitoring (SHM) system for a civil infrastructure is the combination of equipment adequate to permanently characterize its actions and responses (or just the responses) with processing tools that transform the acquired data into relevant information, which should permit to assess the structural performance of the instrumented elements. In this context, it is very important to develop systems based on the permanent acquisition of data and on its online automatic processing to provide valuable information to operators and owners of relevant infrastructures.

Nowadays, an increasing interest in permanent monitoring of the structural behaviour of crucial civil infrastructures, such as bridges, has been observed. This is due to the need of controlling a huge number of structures that are reaching their critical age and also to the necessity of validating the performance of new structures with high levels of complexity. Moreover, recent technological advances have contributed to make the installation and operation of permanent monitoring systems more practical and economical and permit a very efficient transmission and processing of the recorded data.

All over the world there are already several advanced applications of SHM systems on bridges. Trying to cover emblematic examples from different countries documented in available bibliography, the following applications can be mentioned: Tsing Ma Bridge in Hong Kong (Wong 2004), Akashi Kaikyo Bridge in Japan (Abe and Fujino 2009), Seohae
Bridge in Korea (Koh et al. 2009), Confederation Bridge in Canada (Londono 2006), Commodore Barry Bridge in the United States (Aktan et al. 2003) and Oresund Bridge in Denmark (Peeters et al. 2003). A large number of other bridge monitoring systems are described in (Boller et al. 2009) and Ko and $\mathrm{Ni}$ (2005) present a brief synthesis of the monitoring systems installed in twenty Chinese bridges. Despite the greater attention devoted to bridge applications, SHM systems have also been implemented in other civil engineering structures. Brownjohn (2007) describes relevant SHM applications in dams, offshore structures, buildings, towers, nuclear installations and tunnels. Descriptions of further examples are also available in the databases of SAMCO (www.samco.org) and SHMII (www.ishmii.org) associations.

The implementation of well designed structural health monitoring systems permits to: (1) check design assumptions, specially when novel materials or structural systems are adopted; (2) verify serviceability limits and provide alerts when pre-defined thresholds are overcome (for instance the occurrence of strong winds may impose the closure of a bridge); (3) evaluate the structural condition and detect possible damages at an early stage; (4) provide information for safety assessment immediately after extreme events such as earthquakes; (5) provide useful data for the planning of inspections and rehabilitation or maintenance operations; (6) evaluate the effectiveness of maintenance, retrofit or repair works; and (7) obtain large amounts of in-situ data useful to better 
understand load-response mechanisms and consequently improve the design codes.

Despite the already considerable number of practical applications, a large research effort is still needed to improve the present management and processing capabilities in order to conveniently explore the data collected by the instrumentation and accomplish all the mentioned potential capabilities.

The achievement of the previously presented goals imposes also the integration of several types of sensors to measure diverse phenomena using different working principals and adopting different sampling rates. The most commonly implemented SHM systems can be divided in the following three main components: (1) static monitoring, characterized by the adoption of sampling rates of the order of 1 sample/hour, which aims the characterization of phenomena with a low rate of variation, as for instance the temperature at several points of the structure, the relative displacements at the joints and the evolution of displacements, rotations and strains motivated by concrete creep and shrinkage or settlements at the supports; (2) dynamic monitoring, which encompasses the characterization of the structural response to dynamic loads, such as wind, traffic or earthquakes, the evaluation of the levels of vibration for verification of serviceability limit states, the characterization of the time evolution of the structure modal parameters, the characterization of the level of cable vibrations and of the corresponding axial forces through the use of the vibration chord theory; and (3) durability monitoring, which aims the evaluation of the corrosion of steel elements or of the reinforcement of concrete structures due to chloride contamination or carbonation (FIB 2003). Some monitoring systems are also complemented by weather stations for characterization of the environmental factors, weigh-in-motion systems to measure traffic loads and video cameras. The data collected by each monitoring component has to be processed by specific algorithms, but then it is very important to analyse and correlate the achieved results in order to provide to operators and owners only a synthesis of the most relevant outputs from the structural behaviour point of view.

This paper is focused on the use of the operational modal analysis algorithms to process acceleration time series collected by permanent dynamic monitoring systems, so that the recorded data is transformed into information from which the structural performance of the instrumented system can be inferred. The followed procedure is described in the next section. Then, a software package that integrates all the tools needed to follow the procedure described in section 2 is presented. Afterwards, it is described the application of the proposed software in a monitoring program developed in a concrete arch bridge. Finally, the main conclusions of the paper are systematized.

\section{STRUCTURAL HEALTH MONITORING} BASED ON MODAL PARAMETERS

As reported in (Sohn et al. 2002), there exist several alternatives for the features to be extracted from dynamic measurements. However, in the case of civil structures monitored under normal operation, only the features that can be extracted from the responses of the dynamic system to ambient excitation are adequate. Output-only modal analysis techniques permit the identification of the structure modal parameters under operational conditions and therefore, the estimated natural frequencies, mode shapes and modal damping ratios can be adopted as "fitness for purpose" or damage indicators.

For the post-processing of the identified modal parameters, a large number of damage detection algorithms are mentioned in the literature reviews (Doebling et al. 1996; Sohn et al. 2002). These are organized in four levels: (1) detection of damage, (2) localization of damage, (3) quantification of damage, and (4) prediction of the remaining life of the structure. With a permanent dynamic monitoring system it is difficult to go beyond level 1, because in order to keep the monitoring system cost tolerable, generally, only a limited number of points are usually measured. Still, the main goal of a monitoring system is to provide an alert that should then trigger more detailed investigations that may then reach level 4. For the purpose of damage detection, one of the important advantages of using modal parameters is their global nature, which means that damage can be detected (but not localized or quantified) without measuring points in the vicinity of the damaged zone.

The present work is focused on the analysis of the time evolution of modal parameters with the goal of detecting anomalous variations that might be caused by the occurrence of damage. As damages are normally associated with a stiffness reduction, this necessarily implies abnormal reductions of the natural frequencies and therefore this is the modal parameter more commonly used for damage detection. Accurately identified mode shapes can be adopted for the localization of damage only if a quite dense measuring grid is used. The modal damping ratios might be also sensitive to damage but the higher uncertainty usually associated with their estimates makes more difficult the identification of small variations. Besides the detection/localization of damage, modal parameters estimated during normal operation can be also used to better understand some phenomena such as the influence of the wind characteristics on natural frequencies and modal damping ratios or the dependency of modal damping ratios with vibration levels.

The characterization of the time evolution of modal parameters is only possible with algorithms capable of automatically estimate these parameters 
from acceleration time series continuously acquired by permanent dynamic monitoring systems. This can be successfully accomplished by recently developed algorithms (Amador et al. 2011; Magalhães et al. 2009). Then, as the identification of very small structural changes is aimed to permit the detection of damages in an early phase of development, the minute effects of for instance the temperature or the traffic intensity over a bridge on the estimated natural frequencies have to be minimized. Therefore, monitoring software based on operational modal analysis has to include three components: (i) automatic identification of modal parameters, (ii) elimination of environmental and operational effects on modal parameters, (iii) calculation of a suitable index to flag relevant frequency shifts. These steps are illustrated in Figure 1 using results from the monitoring application that will be present in section 4 . The theoretical background of some tools that can be adopted to accomplish tasks (ii) and (iii) is presented in reference (Magalhães et al. 2011). The integration of the three steps in a monitoring software is detailed in the next section.

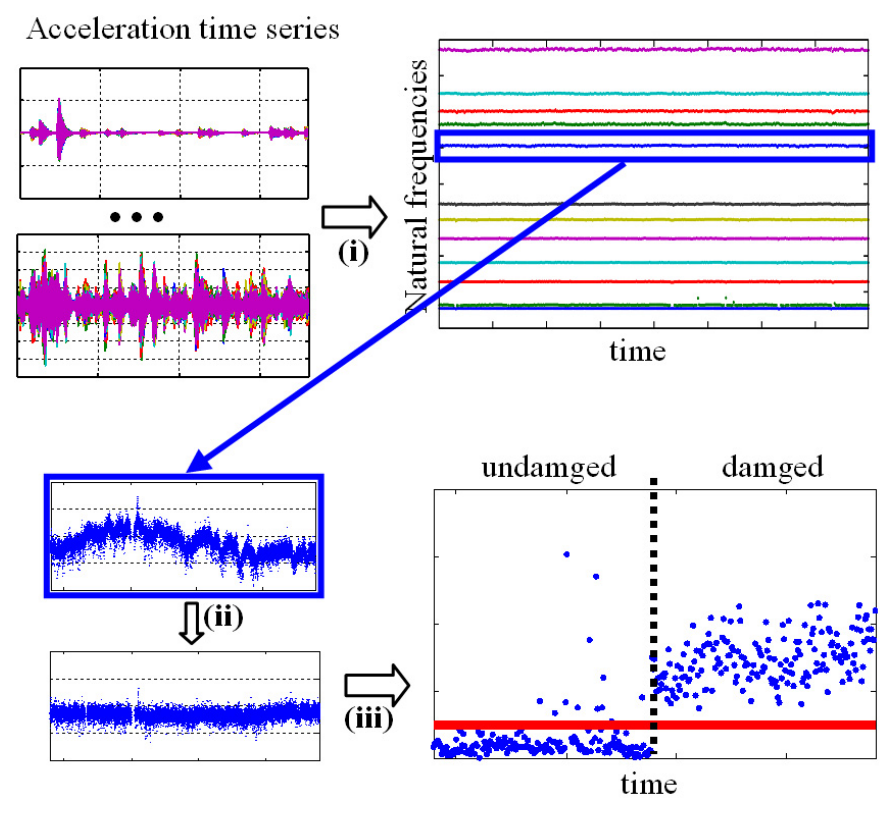

Figure 1. Main processing steps of a vibration-based health monitoring system.

\section{DYNAMO SOFTWARE}

The need for the development of software to efficiently extract useful information from data collected by Dynamic Monitoring Systems is evidenced by the huge difference between the number of bridges with Monitoring Systems and the number of publications showing a clear and useful interpretation of the results provided by those systems. So, the DynaMo software was developed to overcome this need.

The DynaMo was programmed in Matlab, in order to take profit from previously develop identifica- tion algorithm and from the tools included in the programming environment. Then, with the goal of making it flexible, it was compiled to become a standalone application that can be deployed in any computer with any operating system without the need to pre-install Matlab.

The raw data processing can be either processed at the instrumented structure by the same computer that is responsible for the data acquisition or transferred to another computer placed in a different location, where the processing is to be performed. In any case, the data has to be processed right after being acquired or transferred, so that timely alerts can be provided to the infrastructure owner or operator. The quality of nowadays internet services makes feasible the second option, which permits to adopt at the remote site a simpler and more robust computer, and therefore less prone to problems.

The software package is essentially composed by three components: the main processing routines (detailed in section 3.1), a graphical user interface (detailed in section 3.2) and a watchdog software.

This last component is very important to guarantee that the monitoring software is always 'alive'. Since, its function is to keep the DynaMo running it was called Wheel.

\subsection{Processing routines}

The DynaMo software is continuously running in a computer that can be placed in any location with an internet connection. At the instrumented structure the monitoring equipment should create data files a predefined rate (for instance each 30 minutes). After the creation of each new data file at the remote site, DynaMo executes the following tasks:

- download of the most recent data file;

- archive of the original data in a database;

- pre-processing of data to eliminate the offset and, if necessary, apply filters and reduce the sampling frequency;

- characterization of the signal amplitude by its maxima and root mean square values;

- processing of data for automatic identification of modal parameters using three different identification algorithms: Frequency Domain Decomposition (FDD), Covariance driven Stochastic Subspace Identification (SSI-COV) and polyLeast Squares Complex Frequency Domain (pLSCF);

- post-processing of the identified natural frequencies to minimize the effects of the environmental and operational factors.

- construction of control charts for the identification of abnormal values;

- creation of a database with all the results.

Concerning to the raw data files, a considerable amount of memory is required to keep them in a da- 
tabase and, therefore, these original information can either be discarded or optionally kept for future research purposes.

The pre-processing tools include several alternative filters (low-pass, high-pass or band-pass) to select the frequency range of interest for the subsequent analysis. This can be followed by a resampling in order to reduce the number of points of the time series and so make the subsequent processing more efficient. After this, the maxima and the root mean square values of all the collected time series are characterized. This permits the continuous verification of serviceability limit states, especially relevant for instance in footbridges. In section 4 , it will be shown that the signal amplitude is also important to explain fluctuations of modal parameters.

The automatic identification of modal parameters is the most important task of the DynaMo software. Three different state-of-the-art algorithms were implemented, which gives to the user the freedom to apply just one or to simultaneously use the three and then select the results with higher quality. These algorithms present some parameters that have to be tuned for each new application. Therefore, the software contains routines to perform some preliminary offline processing with several intermediate results that facilitate the adjustment of the parameters of the algorithms for automatic identification. The identification of modal parameters is followed by a procedure that links the new set of modes with the ones identified in previous setups, explained in (Magalhães et al. 2009).

The identified modal parameters can then be post-processed by routines that minimize the effect of the environmental and operational factors. The DynaMo permits the use of multivariate regression models or/and the adoption of models derived from principal components analyses. As explained in (Magalhães et al. 2011), these models should be established using the time evolution of natural frequencies under a significant range of variation of environmental and operational factors. Therefore, their application only starts one year after the beginning of the monitoring program. After each complete year, the models can be improved to incorporate a larger range of variation of the factors that influence the modal parameters.

Afterwards, the outputs of the previous postprocessing are synthesized in control charts that permit the detection of abnormal values that may then trigger alarms.

All the important results of the data processing are stored in a database that follows the organization illustrated in Figure 2. There is a folder for each month that contains one file for each day. These daily files (with the extension *.mat) contain matrices with different sets of results: maxima, root mean square values, modal parameters identified by all the applied identification techniques, etc. Adopting as example the data of the Infante D. Henrique Bridge, it is interesting to observe that the 48 files associated with each day, with more than $144 \mathrm{MB}$, are transformed into information that can be stored in a file with less than $700 \mathrm{~KB}$.

\begin{tabular}{|c|c|c|}
\hline 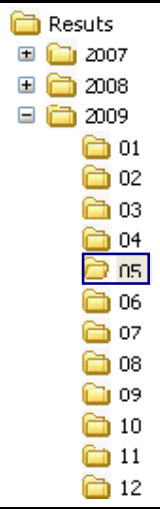 & 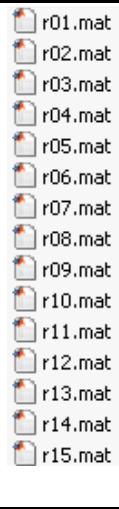 & $\begin{array}{l}692 \mathrm{~KB} \\
692 \mathrm{~KB} \\
692 \mathrm{~KB} \\
693 \mathrm{~KB} \\
693 \mathrm{~KB} \\
692 \mathrm{~KB} \\
692 \mathrm{~KB} \\
692 \mathrm{~KB} \\
693 \mathrm{~KB} \\
692 \mathrm{~KB} \\
692 \mathrm{~KB} \\
692 \mathrm{~KB} \\
692 \mathrm{~KB} \\
692 \mathrm{~KB} \\
693 \mathrm{~KB}\end{array}$ \\
\hline
\end{tabular}

Figure 2. Organization of the database with the files that contain the outputs of the processing.

The DynaMo has the status window presented in Figure 3 that displays the operations that are being performed by the software. Besides the data download and processing, for the case of the Infante D. Henrique Bridge, there is an extra operation that is needed to obtain the temperature readings. As the temperature at several structural elements is measured by a different monitoring system, the temperature values are not included in the downloaded data files and therefore, it was necessary to develop an extra routine to continuously extract the temperature values stored in the database of the static monitoring system.

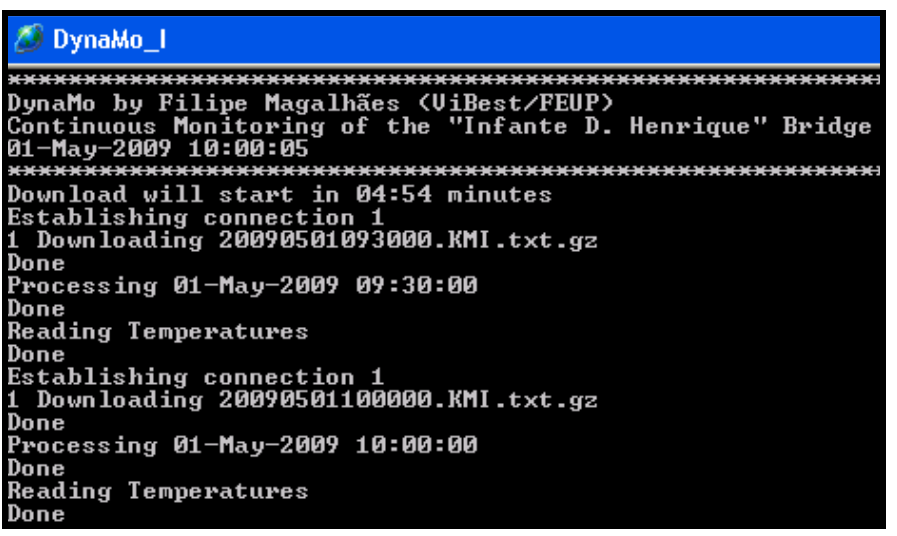

Figure 3. Status window of the DynaMo software.

The window is completed by the one associated with the Wheel software presented in Figure 4. This watchdog software verifies if the DynaMo is downloading the files at the time it is programmed to, and monitors the downloading process. If the Wheel finds fails (these sometimes occur due to sporadic problems in the internet connection), then it means that the DynaMo is not working properly and consequently it is restarted. 


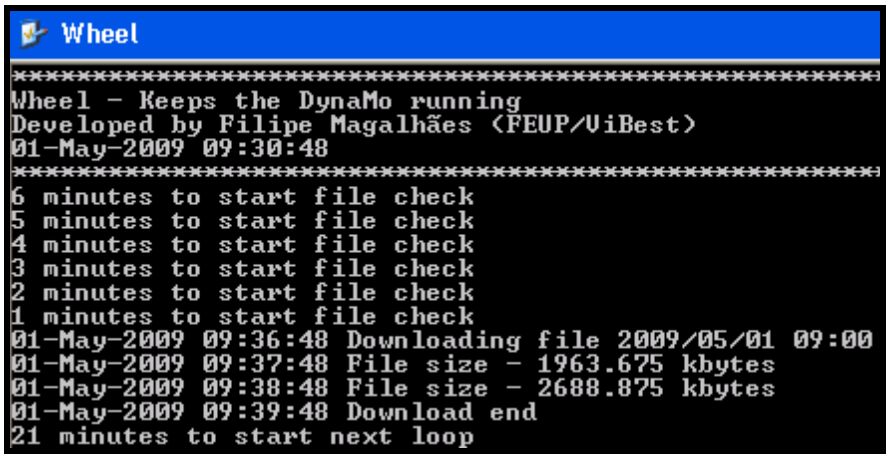

Figure 4. Status window of the Wheel software.

\subsection{Graphical interface}

In order to facilitate the access to the database with the results, several routines were implemented to create various types of graphics. Later, these were integrated in a Graphic User Interface (GUI), named DynaMo Viewer (Diord et al. 2009). These were also programmed in Matlab and then compiled to become a windows standalone application. Figure 5 presents the main windows of this GUI customized for the application in the Infante D. Henrique Bridge (described in section 4). It is essentially composed by a table in the form of a calendar that displays all the available setups. After the selection of a time period, which can vary from one day to several months, it is possible to have access to a list of setups collected during that period and obtain plots with the time evolution of several parameters, such as the modal parameters estimated with the selected identification algorithm, as presented in Figure 6.

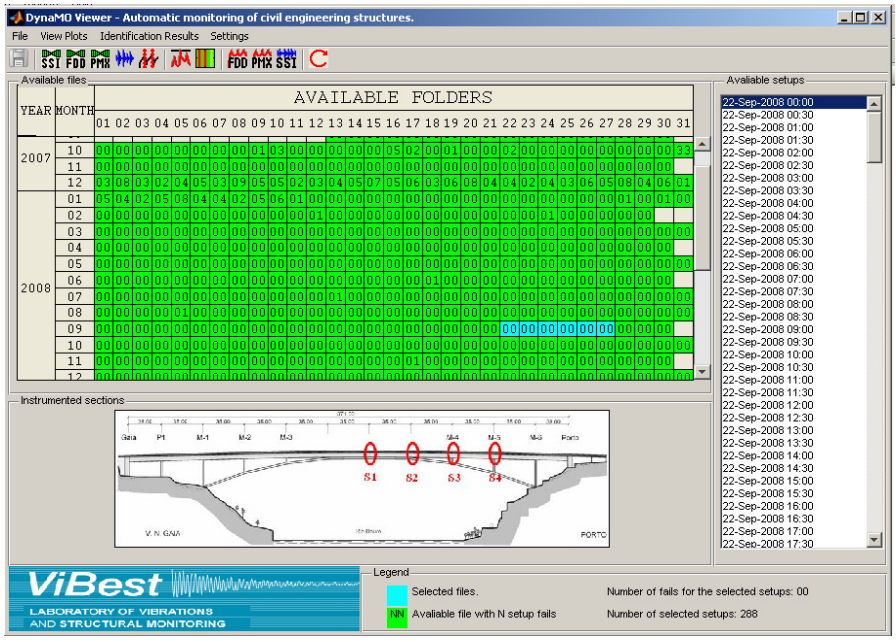

Figure 5. Main window of the DynaMo Viewer software.

Figure 7 shows all the windows that can be open with the DynaMo Viewer interface. Besides the one presented in Figure 6, these include plots with the time evolution of the maxima and root mean square values of the acceleration time series, colour maps with the time evolution of the frequency content of the measured structural responses, plots with the correlation between temperature and modal parameters, animations of the mode shapes identified in a selected setup. Further detail are presented in reference (Amador et al. 2009).

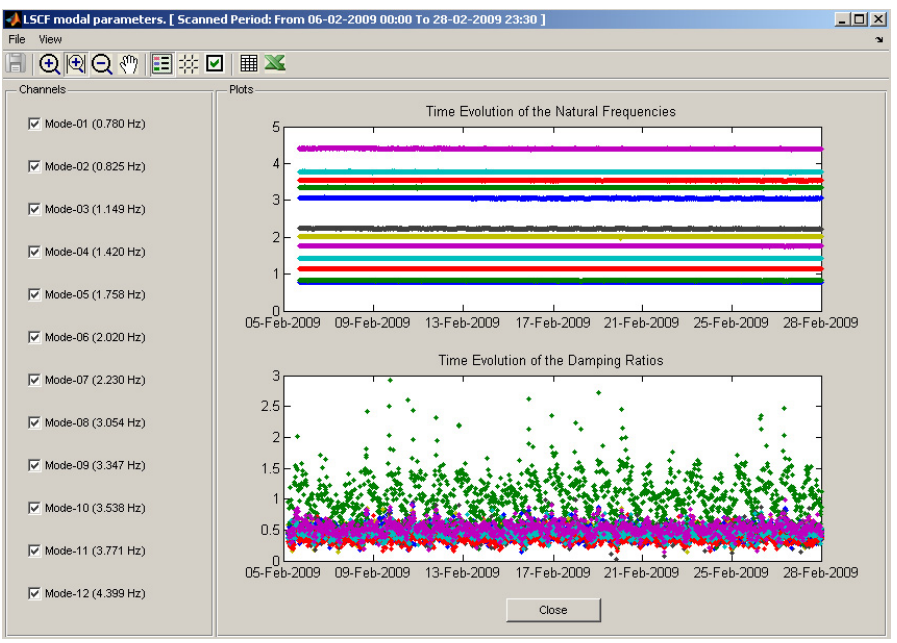

Figure 6. One set of the plots produced by the DynaMo Viewer software (time evolution of 12 natural frequencies and respective modal damping ratios).

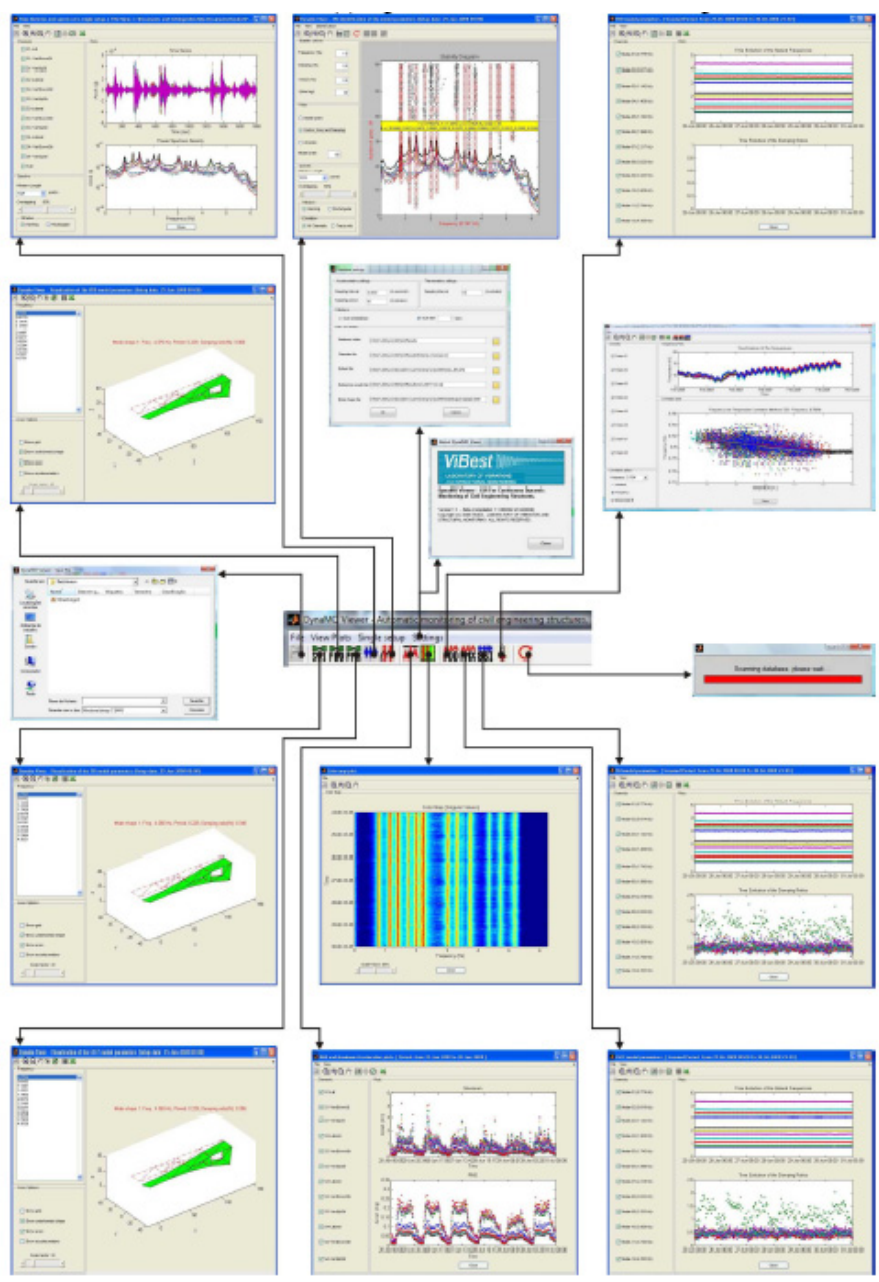

Figure 7. Overview of the DynaMo Viewer organization (Amador et al. 2009).

\section{APPLICATION}

In this section the use of the DynaMo software package is illustrated with an application on a very relevant bridge of the Portuguese road infrastructure. 


\subsection{Description of the bridge and monitoring system}

The Infante D. Henrique Bridge, over the Douro River, was open to traffic in 2004 to link the cities of Porto and Gaia, located at the north of Portugal.

The bridge is composed of a rigid prestressed concrete box girder, $4.50 \mathrm{~m}$ deep, supported by a shallow and thin reinforced concrete arch, $1.50 \mathrm{~m}$ thick (Figure 8). The arch spans $280 \mathrm{~m}$ between abutments and rises $25 \mathrm{~m}$ until the crown. In the $70 \mathrm{~m}$ central segment, arch and deck join to define a box girder $6 \mathrm{~m}$ deep. The arch has constant thickness and its width increases linearly from $10 \mathrm{~m}$ in the central span up to $20 \mathrm{~m}$ at the springs (Adão da Fonseca and Millanes Mato 2005).

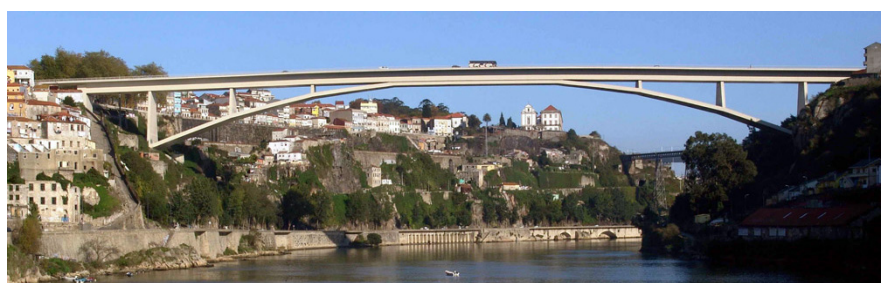

Figure 8. Infante D. Henrique Bridge.

The dynamic monitoring system of the Infante D. Henrique Bridge is essentially composed by 12 force balance accelerometers, 2 digitizers and an internet router, which are installed inside the deck box girder and distributed along the bridge according to the scheme presented in Figure 2. Three sensors equip each section: one to measure the lateral acceleration and two for the vertical acceleration at the downstream and upstream sides. These are force balance accelerometers (FBA ES-U2) from Kinemetrics. Each digitizer (www.Q330.com) allows the connection of six dynamic channels, is based on 24-bit analogue-to-digital converters and permits simultaneous telemetry of the acquired data to a central site and a link to a local recording unit. In the present installation, the digitizers were placed at sections S2 and S4 (Figure 9). Digitizers located at sections S2 and S4 are connected to each other by an Ethernet cable. Another Ethernet cable links section S4 and the router that is the interface between the local network and the Internet. Synchronization between digitizers is achieved with two GPS antennas and receivers that allow continuous update of the internal clocks of both units.

The data produced by the two digitizers becomes available at FEUP in the form of ASCII files with as many columns as the number of sensors, containing acceleration time series sampled at a predefined rate and time length. In this installation, a sampling frequency of $50 \mathrm{~Hz}$ and a length of $30 \mathrm{~min}$ were selected.

This dynamic monitoring system is complemented by an independent static monitoring system (performing six acquisitions per hour) that was in- stalled in the bridge during construction, comprising strain gages, clinometers and temperature sensors (http://www.kinesia.net/). In particular, the temperature sensors embedded in the concrete (see Figure 9) were crucial for the studies presented in the remaining sections.

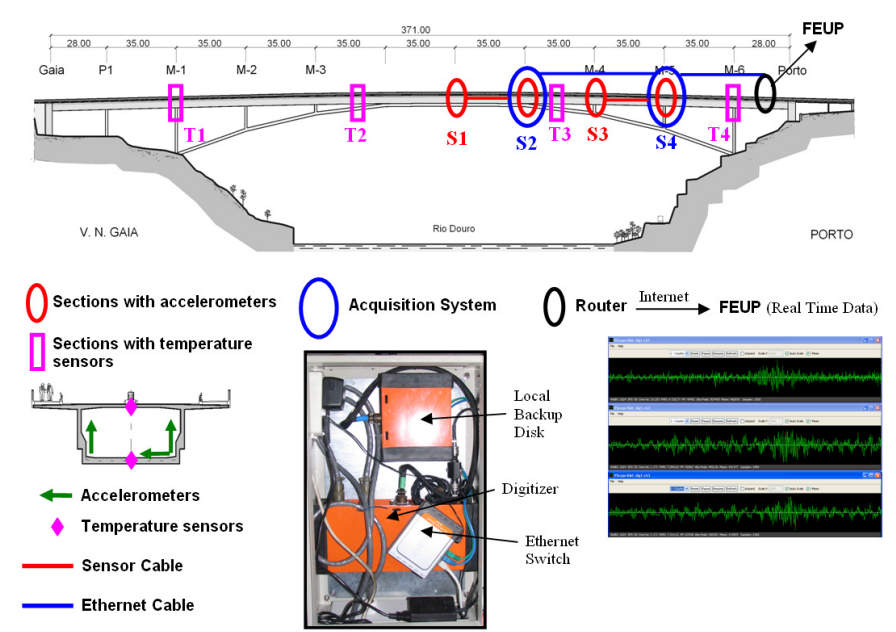

Figure 9. Scheme of the Dynamic Monitoring Hardware and position of the temperature sensors.

\subsection{Main results}

The Dynamic Monitoring system of the Infante D. Henrique Bridge is working since the $13^{\text {th }}$ of September of 2007. Therefore, a database with the variation of the bridge modal parameters identified with the three implemented methods (FDD, SSI$\mathrm{COV}$ and p-LSCF) during more than four years is available. All the methods permitted the track of the bridge first 12 vibration modes, but since previous comparison studies have proven the p-LSCF method provided the best results (Magalhães 2010), in the present section, only the estimates provided by this method are presented,

Figure 10 presents the evolution of the bridge first 12 natural frequencies during the first monitoring year. Since only on half of the bridge is instrumented, with the monitoring system, it is not possible to obtain a complete picture of the modes shapes, so these were previously identified with an ambient vibration test. Some of the mode shapes associated with the natural frequencies analysed in Figure 10 are presented in Figure 11.

In a first analysis of Figure 10, it seems that the natural frequencies remain constant over time. However, zooms around each one clearly demonstrate daily and annual fluctuations. One of these zoom is presented in Figure 12, to illustrate the time evolution of the natural frequency of the second vertical bending mode over the last four years. It is clear a frequency increase over each winter and a decrease during each summer (annual variation of about $0.01 \mathrm{~Hz}$ due temperature variations from 5 to $35^{\circ} \mathrm{C}$ ). Besides this, it is also observed a small increase of the average frequency value over these four years 
that might be explained by the concrete hardening. Similar plots are observed for the other bridge natural frequencies.

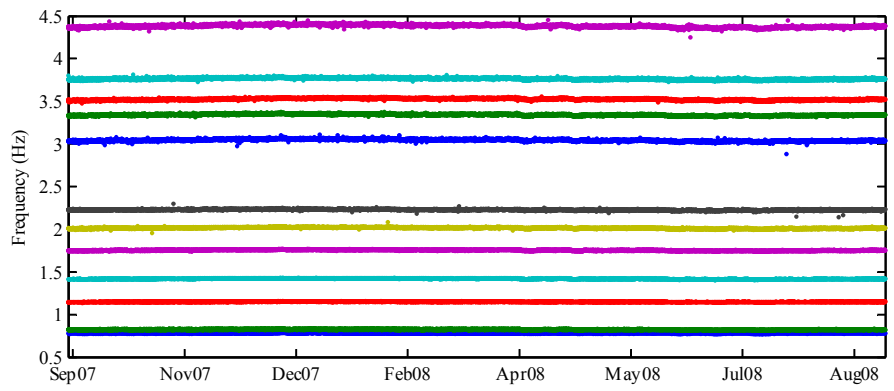

Figure 10. Time evolution of the bridge 12 first natural frequencies from 13/09/2007 to 13/09/2008.

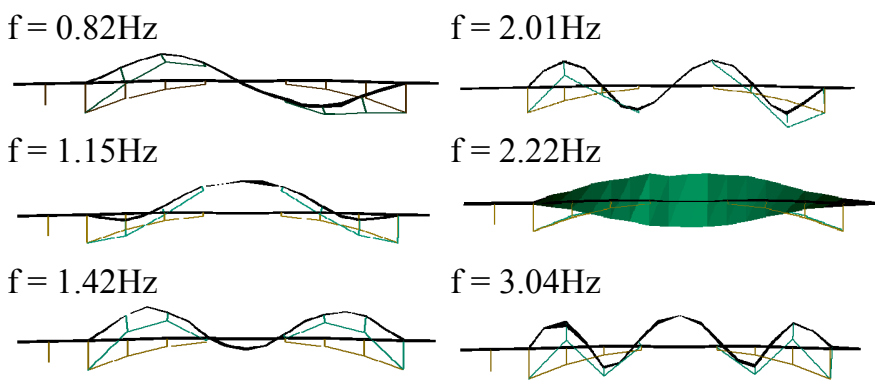

Figure 11. First 6 vertical bending/torsion mode shapes.

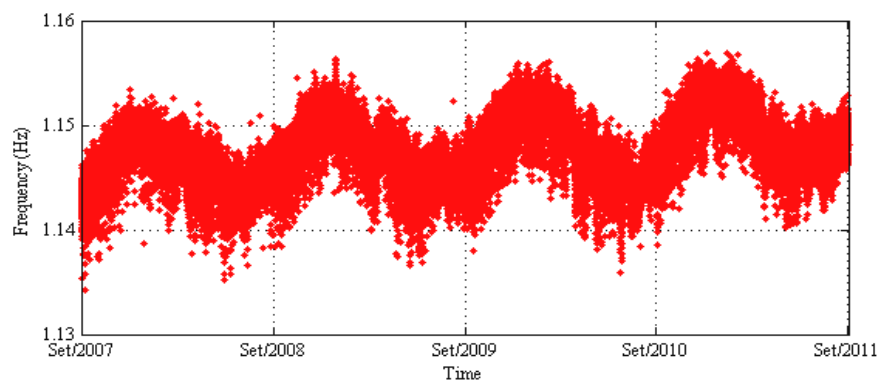

Figure 12. Time evolution of the natural frequency of the second vertical bending mode from 13/09/2007 to 13/09/2011.

Another factor with relevant influence on the natural frequencies is the amplitude of the bridge vibration, which is essentially related with the traffic intensity. This dependency is characterized in Figure 13 for the first bending mode, during the first week of March and using the root mean square value (RMS) as a measure of the vibration intensity. The second mode is the one from the twelve analysed modes that experiences higher daily fluctuations. This night be explained by the relevant movement in the longitudinal direction associated with this mode, that is influenced by the friction forces of the abutment bearings. For low vibration amplitudes the bearings behave has a fix connection, whilst for large amplitudes the friction forces are overcome and, as a consequence, the natural frequency decreases (it is worth noting that the scale associated with the RMS is inverted).

In order to better understand the effect of the traffic over the bridge on its modal parameters, an average daily evolution of the natural frequencies and modal damping ratios was evaluated. This was ob- tained averaging the modal parameters of all working days of November 2007 associated with the same half an hour. Figure 14 shows the results for all the modal damping ratios of the analyzed modes and for the natural frequency of the first mode. It is observed that all the modal damping ratios show a peak in the period from 7:30 to 10:00 (morning rush hour) and a less pronounced increase in the period from 6:00 to 20:00 (period with higher RMS values, see Figure 13), both effects being enhanced in the second mode (represented by the green line). This happens because at the bridge end, on the Porto side, there are traffic lights that induce traffic jams over the bridge at the morning rush hour. The increase of the damping, which consistently occurs at the same day period, is certainly motivated by the dynamic interaction between the bridge and the vehicles stopped over the bridge. The effects of the traffic jams on the natural frequencies are less prominent, but still observable due the high accuracy achieved in the modal identification. In the case of the natural frequency of the first mode (lateral mode), at the morning rush hour it is observed a sudden decrease of approximately $0.003 \mathrm{~Hz}$, which can only be explained by the extra mass of the cars over the bridge. The same phenomenon is also observable on other modes.

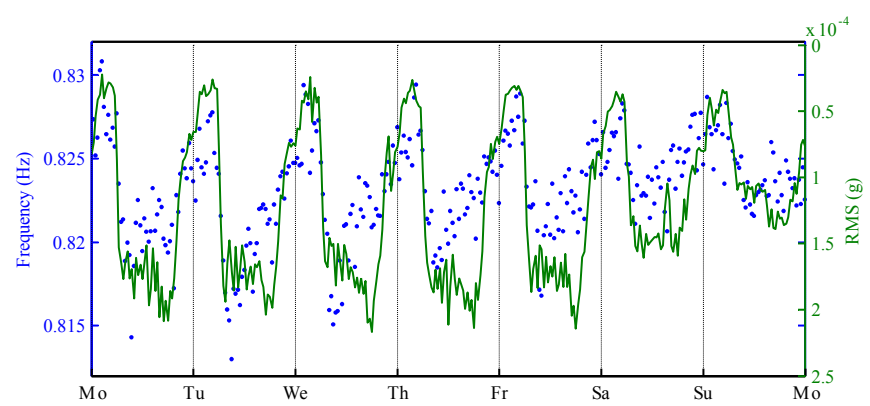

Figure 13. Natural frequency of the first bending mode $v s$ vibration amplitude.

After studying the variation of the modal parameters due to the structure normal operation during a reference period of about one year, it is possible to develop statistical models, using regressions and principal component analysis, that reduce these effects and therefore obtain time series of corrected natural frequencies (Magalhães et al. 2011).

Then, considering all the structure natural frequencies under observation, a control chart can be constructed to permit the identification, in just one plot, of small frequencies shifts that might be due to the eventual appearance of damages.

Figure 15 shows such control charts for the bridge under analysis. The first one is associated with the processing of the real data and so the majority of the points are below the control line (in red) indicating that there are no damages. The other one is associated with data that was manipulated to include the effect of a small damage (reduction of $10 \%$ 
in the inertial of a deck segment with $10 \mathrm{~m}$ which produced frequency variations lower then $0.20 \%$ ). After the instruction of damage all the points of the control chart lay above the control line indicating an abnormal change of the natural frequencies (Magalhães et al. 2011).
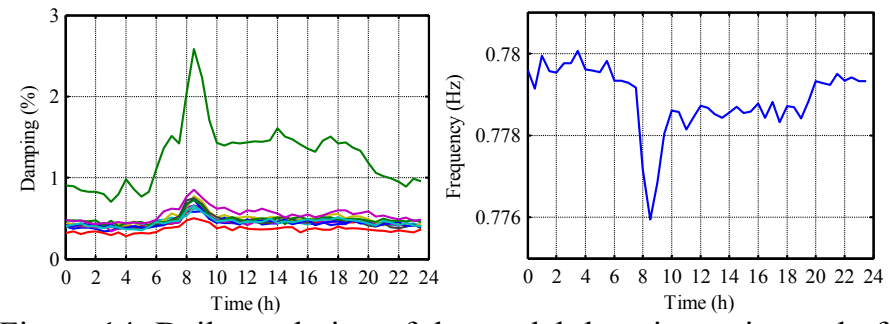

Figure 14. Daily evolution of the modal damping ratios and of the natural frequency of the first lateral bending mode.
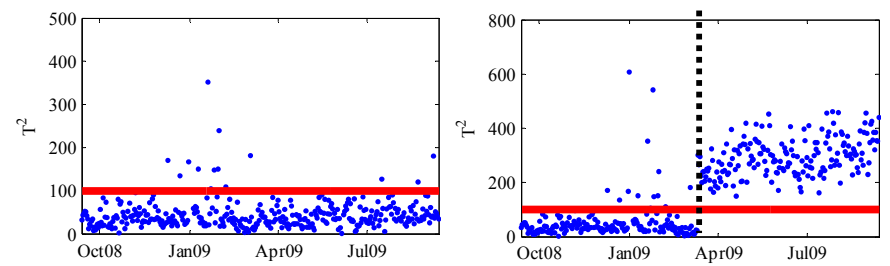

Figure 15. Control chart without damage and with the simulation of damage after the vertical dashed line.

\section{CONCLUSIONS}

This paper presented the DynaMo software. This manages the data collected by dynamic monitoring systems, processes it online using innovative algorithms and produces outputs that permit the detection of abnormal variations that might be associated with damages.

The application on the Infante D. Henrique Bridge demonstrated that the tools included in the DynaMo software permit a very accurate identification of the structure modal parameters, enabling an accurate quantification of the effects produced by temperature and traffic intensity. After the analysis of a reference period, it is possible to eliminate the variation of the modal parameters associated with the normal operation of the structure and detect small variation on the natural frequencies, permitting the detection of damages when they are still in an early phase of development.

\section{ACKNOWLEDGEMENTS}

The authors would like to acknowledge: (1) all the supports provided by the Portuguese Foundation for Science and Technology (FCT) to CEC/ViBest at FEUP for the development of research in the area of Long-Term Dynamic Monitoring, in particular the R\&D Project PTDC/ECM/109862/2009, and the $\mathrm{PhD}$ Scholarship provided to the second author (SFRH/BD/44291/2008); (2) the support provided by the bridge designer, Prof. Adão da Fonseca, and by the bridge owner, Metro do Porto.

\section{REFERENCES}

Abe, M.; Fujino, Y. (2009) Bridge Monitoring in Japan. Encyclopedia of Structural Health Monitoring, C. Boller, F. Chang, and Y. Fujino, eds., Vol. 5, John Wiley \& Sons.

Adão da Fonseca, A.; Millanes Mato, F. (2005) Infante Henrique Bridge over the River Douro, Porto, Portugal. Structural Engineering International, 15(2), 85-87.

Aktan, A. E.; Catbas, F. N.; Grimmelsman, K. A.; Pervizpour, M. (2003) Development of a Model Health Monitoring Guide for Major Bridges. Drexel Intelligent Infrastructure and Transportation Safety Institute.

Amador, S.; Magalhães, F.; Caetano, E.; Cunha, A. (2011) Analysis of the influence of environmental factors on modal properties of the Braga Stadium suspension roof. In Proceedings of EVACES, Varenna, Italy.

Amador, S.; Magalhães, F.; Cunha, Á. (2009) DynaMo Viewer User's Guide. Report ViBest/FEUP.

Boller, C.; Chang, F.-K.; Fujino, Y. (2009) Encyclopedia of Structural Health Monitoring. John Wiley \& Sons

Brownjohn, J. M. W. (2007) Structural Health Monitoring of Civil Infrastructures. Philosophical Transactions of The Royal Society A, 365(1851), 589-622.

Diord, S.; Magalhães, F.; Cunha, Á. (2009) DynaMo Viewer User's Guide. Report ViBest/FEUP.

Doebling, S. W.; Farrar, C. R.; Prime, M. B.; Shevitz, D. W. (1996) Damage Identification and Health Monitoring of Structural and Mechanical Systems from Changes in their Vibration Characteristics: a Literature Review. LA-13070-MS, Los Alamos National Laboratory, Los Alamos.

FIB (2003) Monitoring and Safety Evaluation of Existing Concrete Structures FIB Bulletin 22.

Ko, J. M.; Ni, Y. Q. (2005) Technology developments in structural health monitoring of large-scale bridges. Engineering Structures, 27(12), 1715-1725.

Koh, H.-M.; Lee, H.-S.; Kim, S.; Choo, J. F. (2009) Monitoring of Bridges in Korea. Encyclopedia of Structural Health Monitoring, C. Boller, F. Chang, and Y. Fujino, eds., Vol. 5, Chapter 124, John Wiley \& Sons

Londono, N. A. (2006) Use of Vibration Data for Structural Health Monitoring of Bridges. PhD Thesis, Carleton University, Ottawa.

Magalhães, F. (2010) Operational Modal Analysis for Testing and Monitoring of Bridges and Special Structures. PhD Thesis, Faculty of Engineering of the University of Porto.

Magalhães, F.; Cunha, Á.; Caetano, E. (2009) Online automatic identification of the modal parameters of a long span arch bridge. Mechanical Systems and Signal Processing, 23(2), 316-329.

Magalhães, F.; Cunha, Á.; Caetano, E. (2011) Vibration based Structural Health Monitoring of an arch bridge: from automated OMA to damage detection. Mechanical Systems and Signal Processing, doi:10.1016/j.ymssp.2011.06.011.

Peeters, B.; Couvreur, G.; Razinkov, O.; Kundig, C.; Van der Auweraer, H.; De Roeck, G. (2003) Continuous Monitoring of the Oresund Bridge: System and Data Analysis. In Proceedings of IMAC 21, International Modal Analysis Conference, Kissimmee, Florida, USA.

Sohn, H.; Farrar, R.; Hemez, M.; Czarnecki, J.; Shunk, D.; Stinemates, W.; Nadler, R. (2002) A review of Structural Health Monitoring Literature: 1996-2001. Los Alamos National Laboratory, Los Alamos.

Wong, K.-Y. (2004) Instrumentation and Health Monitoring of Cablesupported Bridges. Structural Control and Health Monitoring, 11(2), 91-124. 